\title{
Significado de la progresión de PSA tras prostatectomía radical. Resultados preliminares
}

\author{
Luján Galán M, Pascual Mateo C, Rodríguez García N, Chiva Robles V, Escalera Almendros C, \\ Angulo Cuesta J, Berenguer Sánchez A.
}

\author{
Hospital Universitario de Getafe. Servicio de Urología. Madrid.
}

Actas Urol Esp. 2006: 30 (4): 353-358

\begin{abstract}
RESUMEN
SIGNIFICADO DE LA PROGRESIÓN DE PSA TRAS PROSTATECTOMÍA RADICAL. RESULTADOS PRELIMINARES.

Objetivos: Ofrecer nuestra experiencia respecto de la historia natural del cáncer de próstata (CaP) que sufre progresión tras la prostatectomía radical (PR), en términos de tiempo hasta la aparición de metástasis o hasta la muerte por esta enfermedad. Identificar los factores clínico-patológicos que pueden condicionar dicha evolución.

Material y métodos: Análisis retrospectivo de 227 pacientes con CaP clínicamente localizado sometidos a PR. Se definió el evento progresión bioquímica (PBQ) como el presentar un PSA postoperatorio de $0,2 \mathrm{ng} / \mathrm{ml}$ al menos tres meses tras la cirugía, excluyendo del análisis los casos con tiempo de seguimiento inferior al mencionado. No se administró tratamiento precoz (radioterapia externa o bloqueo hormonal) en los pacientes incluidos.

Se calculó la supervivencia libre de PBQ a lo largo del tiempo (análisis Kaplan-Meier). Se estudió (análisis uni y multivariante) la capacidad de factores como PSA preoperatorio, score de Gleason en la pieza, presencia de penetración capsular, margen quirúrgico afectado (excluyendo uretral), extensión extracapsular, presencia de ganglios positivos, y afectación de vesículas seminales, para predecir la recurrencia.

Por último, para el grupo de pacientes que presentaron PBQ, calculamos la probabilidad de permanecer libre de metástasis a distancia a lo largo del seguimiento, así como la función de supervivencia cáncer-específica.

Resultados: Un total de 208 registros fueron finalmente incluidos en el estudio. La mediana de edad fue de 61 años. Un total de 47 (22,6\%) presentó estadio patológico extracapsular. La mediana de seguimiento fue de 35,8 meses. Un total de 49 (23,6\%) presentaron PBQ. La probabilidad de permanecer libre de PBQ fue de $79,9 \%$ y $67,4 \%$ a los 2 y 5 años, respectivamente.

Tan sólo tres factores fueron identificados por el modelo multivariante como predictores independientes de recurrencia: un PSA preoperatorio >= $10 \mathrm{ng} / \mathrm{ml}$ (hazard ratio -HR- 3,03), score de Gleason en la pieza quirúrgica entre 8 y 10 (HR 3,42), y el hallazgo de penetración capsular (HR 2,17).

De los pacientes que presentaron PBQ, 16,3\% desarrolló metástasis a distancia. Las probabilidades de permanecer libre de metástasis tras la PBQ fueron calculadas en $97,7 \%$ y $86,9 \%$ a los 2 y a los 5 años respectivamente (mediana actuarial de 110,8 meses). Sólo 2 individuos fallecieron por CaP por lo que no se llevó a cabo análisis de mortalidad cáncer-específica.

Conclusiones: A pesar de la notable proporción de pacientes con progresión de PSA tras la prostatectomía radical en nuestro entorno, su pronóstico a corto y medio plazo, en términos de desarrollo de metástasis, es aceptable. En cualquier caso, un nuevo análisis será necesario para verificar la evolución a largo plazo de estos pacientes.
\end{abstract}

Palabras clave: Historia natural. Cáncer de próstata. Prostatectomía radical.

\section{ABSTRACT}

THE MEANING OF PSA PROGRESSION AFTER RADICAL PROSTATECTOMY. PRELIMINARY RESULTS.

Objectives: To report our findings regarding to the natural history of prostate cancer (PCa) that shows recurrence after radical prostatectomy (RP), in terms of time to development of metastatic disease and death from PCa. To identify independent predictors of PSA recurrence.

Material and methods: Retrospective analysis of 227 patients with clinically localized PCa who underwent RP. The event PSA recurrence was defined as the presence of a postoperative PSA level of $0,2 \mathrm{ng} / \mathrm{ml}$ or higher at least 3 months after surgery. Hence, cases with shorter follow-up time were excluded from analysis. No adjuvant therapy (radiotherapy or hormonal therapy) was performed in the included population.

Recurrence free survival was calculated during the follow-up period (Kaplan-Meier analysis). Uni and multivariate study was performed in order to assess the ability of factors as preoperative PSA level, Gleason score in surgical specimen, capsular penetration, positive surgical margins (excluding urethral), extracapsular extension, positive pelvic lymph nodes, and seminal vesicle invasion, to predict PSA recurrence.

Finally, we selected the group of patients with PSA recurrence and calculated the probability of being free from distant metastatic disease during the follow-up period. Also, function of disease-specific survival was calculated.

Results: A total of 208 records were finally included in the study. Median age was 61 years. A total of 47 (22.6\%) presented with extracapsular extension. Median follow-up time was 35.8 months, and $49(23.6 \%)$ developed PSA recurrence. Recurrence free survival was $79.9 \%$ and $67.4 \%$ at 2 and 5 years, respectively.

Only three factors were identified with the aid of multivariate analysis as independent predictors of recurrence: preoperative PSA >= 10 ng/ml (hazard ratio -HR- 3.03), Gleason score in surgical specimen 8 or higher (HR 3.42), and the finding of capsular penetration (HR 2.17).

When only patients with PSA recurrence were considered, $16.3 \%$ developed distant metastasis. Probabilities of being free from distant disease after PSA recurrence were $97.7 \%$ and $86.9 \%$ at 2 and 5 years respectively (actuarial median time 110.8 months). Only 2 patients died from PCa, therefore disease-specific mortality analysis was not performed.

Conclusions: Although an important proportion of patients present with PSA recurrence after RP in our setting, the prognosis in term of development of metastatic disease is acceptable in the short-medium term. Anyway, further analysis will be needed to ascertain the evolution of these patients in the long term.

Keywords: Natural history. Prostate cancer. Radical prostatectomy. 
$\mathrm{E}_{\mathrm{p}}^{\mathrm{s}}$ cáncer de próstata (CaP) es la segunda neoplasia más diagnosticada en el varón en nuestro país. La incidencia ajustada a la pirámide de población española en nuestro entorno (área sanitaria 10 de la Comunidad de Madrid) fue de 106,6 nuevos casos/100.000 varones durante el año 2003.

Durante los últimos años, el patrón oro del tratamiento del CaP localizado ha sido la prostatectomía radical (PR). Con esta modalidad se han publicado resultados de supervivencia libre de progresión bioquímica o de PSA (PBQ) a los 5 años entre el 77 y el $80 \%{ }^{1}$. No obstante, ante la presencia de recurrencia, son actualmente controvertidos tanto el beneficio del tratamiento de la misma, como su modalidad y el momento más adecuado para instaurarlo ${ }^{1}$.

En el presente trabajo pretendemos ofrecer nuestra experiencia respecto de la historia natural del CaP que sufre progresión tras la $\mathrm{PR}$, en términos de tiempo hasta la aparición de metástasis o hasta la muerte por esta enfermedad. Asimismo, se identificarán los factores clínicopatológicos que pueden condicionar dicha historia natural.

\section{MATERIAL Y MÉTODOS}

Hemos realizado un análisis retrospectivo empleando nuestra base de datos de 819 pacientes con $\mathrm{CaP}$ diagnosticado en nuestro área entre Enero de 1991 y Marzo de 2005. Para este estudio hemos partido de 227 registros de pacientes sometidos a PR. Dicha intervención fue realizada mediante $\mathrm{PR}$ retropúbica abierta según técnica descrita por Walsh ${ }^{2}$ en toda la serie, con la excepción de 39 casos (los casos más recientes y, por tanto, con menor tiempo de seguimiento) en los que se realizó por vía laparoscópica. En nuestro centro contemplamos la PR únicamente en tumores clínicamente localizados. Hemos definido el evento PBQ como el presentar un PSA postoperatorio de $0,2 \mathrm{ng} / \mathrm{ml}$ al menos tres meses tras la cirugía (por tanto, excluyendo del análisis los casos con tiempo de seguimiento inferior al mencionado), confirmando este hecho al menos en dos determinaciones consecutivas. Según el protocolo vigente en nuestro Servicio, no se administró tratamiento precoz (radioterapia externa o bloqueo hormonal) en los pacientes incluidos, reservándose hasta la aparición de síntomas relacionados o la aparición de metástasis a distancia. Por ello, fueron excluidos los pacientes en quienes, de modo excepcional, fue administrado tratamiento adyuvante.

Inicialmente hemos calculado la probabilidad de permanecer libre de PBQ (supervivencia libre de progresión) a lo largo del tiempo. Para ello hemos empleado un análisis de supervivencia (Kaplan-Meier) $^{3}$.

Posteriormente hemos estudiado una serie de factores clínico-patológicos (edad, PSA preoperatorio, score de Gleason en la pieza de prostatectomía radical, presencia de penetración capsular, margen quirúrgico afectado, extensión extracapsular, presencia de ganglios positivos, y afectación de vesículas seminales) para identificar cuáles son capaces de predecir de modo independiente la aparición de PBQ. Para ello se empleó un análisis univariante mediante curvas Kaplan-Meier y comparación de las mismas mediante test de Log-Rank (Mantel-Haenszel-Cox), así como un modelo multivariante de regresión de Cox.

Por último, seleccionando el grupo de pacientes que presentaron $\mathrm{PBQ}$, hemos calculado la probabilidad de permanecer libre de metástasis a distancia $(\mathrm{M}+)$ a partir del momento del evento $\mathrm{PBQ}$ hasta el final del seguimiento, así como la función de supervivencia cáncer-específica. Para el análisis de estos pacientes, se excluyeron los casos con tiempo de seguimiento nulo desde la PBQ.

El procesamiento de datos y el análisis estadístico se llevó a cabo con los programas Microsoft Access (Microsoft Corp. Chicago IL, USA) y SPSS (SPSS Inc. Chicago IL, USA).

\section{RESULTADOS}

Se partió de los registros de 227 pacientes sometidos a PR en nuestro centro. Un total de 208 fueron evaluables (tiempo de seguimiento igual o superior a los 3 meses tras la PR) y finalmente incluidos para el estudio. La edad de estos pacientes osciló entre los 41 y los 73 años, media 60,9, error estándar (EE) 0,41, mediana 61 años. Un total de $47(22,6 \%)$ pacientes mostró estadio patológico extracapsular. El tiempo de seguimiento de la serie estudiada osciló entre los 3,1 y 152,7 meses, con media de 44,2 (EE 2,34) y mediana de 35,8 meses. 


\section{PROGRESIÓN BIOQUIIMICA O DE PSA}

De los 208 pacientes evaluables, un total de $49(23,6 \%)$ presentaron PBQ en algún momento del seguimiento. En la Figura 1 se detalla la probabilidad de permanecer libre de PBQ según el momento del seguimiento. Dicha probabilidad fue de $79,9 \%$ y de $67,4 \%$ a los 2 y 5 años, respectivamente. La media actuarial del tiempo hasta la aparición de PBQ fue de 87,6 meses (EE $4,6)$ (mediana no calculable). En la Figura 2 se muestra la densidad de probabilidad de PBQ, donde puede observarse que el mayor riesgo de presentar recurrencia tras la PR está presente principalmente durante los 2 primeros años del postoperatorio.

\section{IDENTIFICACIÓN DE FACTORES PREDICTO- RES DE PROGRESIÓN BIOQUIMMICA}

\subsection{Análisis univariante}

En la Tabla 1 se resumen los factores predictores objeto del estudio y su frecuencia de aparición en la población del estudio.

Se encontró una relación univariante significativa entre el presentar un PSA preoperatorio >=10 ng/ml y la aparición de PBQ (Log-Rank

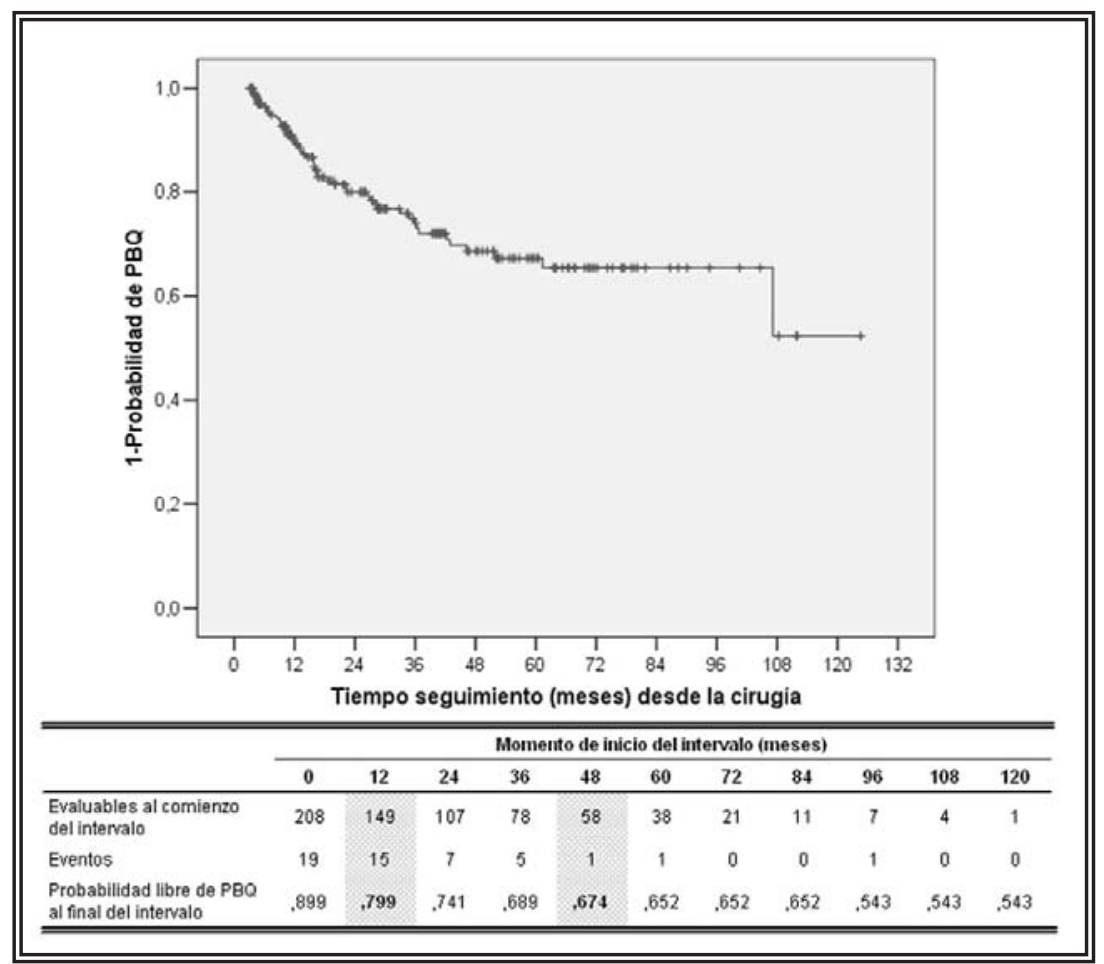

FIGURA 1: Curva Kaplan-Meier que muestra la supervivencia libre de progresión de PSA a lo largo del tiempo en la población seleccionada. $\mathrm{p}<0,001)$. Similares resultados se encontraron para otros factores, como el presentar un score de Gleason pobremente indiferenciado (8-10) en la pieza quirúrgica (Log-Rank $\mathrm{p}<0,001$ ), el presentar penetración capsular (Log-Rank $\mathrm{p}=0,002$ ), margen quirúrgico (excluyendo el uretral) afectado (Log-Rank p=0,019), evidencia de extensión extracapsular (Log-Rank $\mathrm{p}<0,001)$. Por el contrario, la relación entre invasión de vesículas seminales y progresión de PSA no alcanzó rango significativo (Log-Rank $\mathrm{p}=0,082$ ). No se incluyó en el análisis el evento invasión ganglionar (tan sólo dos eventos producidos). Las curvas Kaplan-Meier correspondientes a las mencionadas comparaciones se representan en la Figura 3.

\subsection{Análisis multivariante.}

Tras el modelo multivariante de Cox, tan sólo tres factores fueron identificados como predictores independientes de la aparición de PBQ (Tabla 2): el presentar un PSA preoperatorio $>=10 \mathrm{ng} / \mathrm{ml}$ (hazard ratio $-\mathrm{HR}-3,03$ ), un score de Gleason en la pieza quirúrgica entre 8 y 10 (HR 3,42), y el presentar penetración capsular (HR 2,17).

\section{EVOLUCIÓN TRAS LA PROGRESIÓN DE PSA \\ 3.1. Progresión a enfermedad metastásica.}

Partiendo del grupo con 49 pacientes que presentaron $\mathrm{PBQ}$, hemos excluido los pacientes con tiempo de seguimiento nulo tras la PBQ, por lo que realizamos el análisis con 43 pacientes. De ellos, 7 $(16,3 \%)$ desarrolló metástasis a distancia en algún momento del seguimiento.

El tiempo de seguimiento a contar desde la PBQ osciló entre los 5,5 y los 146,1 meses, media de 48,4 (EE 5,34) y mediana de 36,6 meses. Hemos calculado una probabilidad de permanecer libre de metástasis de $97,7 \%$ y $86,9 \%$ a los 2 y a los 5 años respectivamente (Figura 4). 


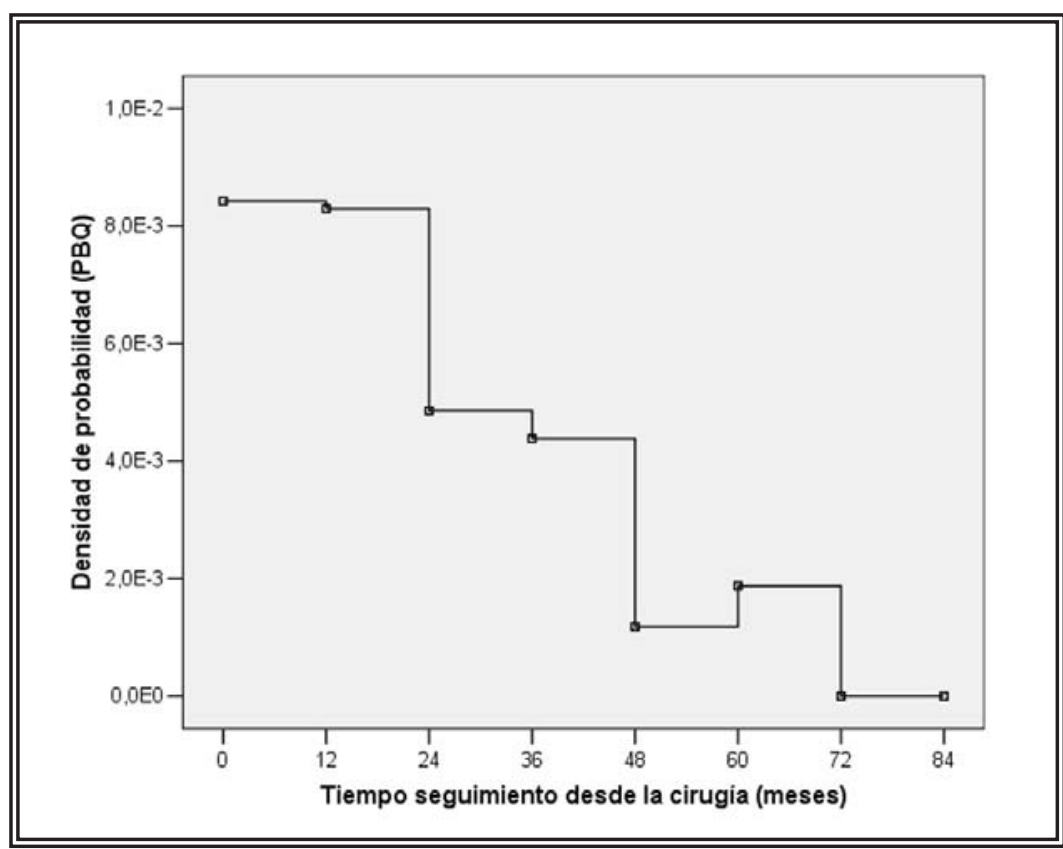

FIGURA 2: Gráfico que muestra la densidad de probabilidad de presentar recurrencia de PSA. Nótese que el mayor riesgo de recurrencia se presenta durante los dos primeros años tras la cirugia.

Tabla 1

Frecuencia de aparición de los factores supuestamente predictores de progresión de PSA en la población seleccionada para el estudio $(n=208)$.

\begin{tabular}{lccc}
\hline & & Frecuencia & Proporción (\%) \\
\hline PSA preoperatorio (ng/ml) & $<4$ & 38 & 18,7 \\
& $>=4 \mathrm{y}<7$ & 80 & 39,4 \\
& $>=7 \mathrm{y}<10$ & 41 & 20,2 \\
& $>=10$ & 44 & 21,7 \\
Score de Gleason & $2-7$ & 179 & 89,1 \\
& $8-10$ & 22 & 10,9 \\
Penetración capsular & & 62 & 29,8 \\
Margen + (cualquiera) & & 72 & 34,6 \\
Margen + (no apical) & & 55 & 26,4 \\
Extensión extracapsular & & 47 & 22,6 \\
pN+ & & 2 & 1 \\
Invasión vesículas seminales & & 13 & 6,3 \\
\hline
\end{tabular}

La media y mediana actuariales del tiempo transcurrido desde la PBQ hasta la aparición de metástasis fueron de 107,4 meses (EE 11,83) y 110,8 meses, respectivamente.

\subsection{Mortalidad por cáncer de próstata.}

En este grupo sólo se registraron 2 fallecimientos por $\mathrm{CaP}$. Por este motivo, hemos preferido no realizar el análisis de supervivencia.

\section{DISCUSIÓN}

En el presente trabajo hemos constatado una probabilidad notable de presentar recurrencia de PSA (32,6\% a los 5 años de la PR). Estos datos son más pesimistas que los encontrados por otros autores ${ }^{4,5}$. Por el contrario, el pronóstico a medio plazo de los pacientes con PBQ de nuestra serie no parece alarmante en términos de desarrollo de metástasis: $85,4 \%$ a los 5 años de la recurrencia, frente al $63 \%$ de supervivencia libre de metástasis tras la PBQ encontrado por Pound et $\mathrm{al}^{4}$. Los tratamientos más recomendados para tratar la progresión de PSA en la actualidad son la radioterapia externa, usualmente recomendada en casos de bajo riesgo (tiempo de doblaje de PSA superior a 10 meses, score de Gleason no pobremente indiferenciado, etc.), y el bloqueo hormonal, recomendado en casos en los que se sospecha que la recidiva no sea local, sino distante (invasión de vesículas seminales, ganglios positivos para tumor en la linfadenectomía, score de Gleason pobremente diferenciado, tiempos de doblaje de PSA cortos, etc). No obstante, el beneficio de estas dos modalidades en estos pacientes, en términos de supervivencia, es controvertido ${ }^{1}$.

En nuestro protocolo, debido a dicha controversia, no contemplamos ninguno de los dos tratamientos mencionados en el paciente asintomático con PBQ aislada. A la vista de los resultados ofrecidos, el pronóstico de estos pacientes sin tratamiento es aceptable a medio plazo tanto para la aparición de metástasis como para el fallecimiento por CaP. Ello parece avalar (a corto y medio plazo) la validez del protocolo que hemos elegido para estos pacientes. No obstante, somos conscientes de que el limitado tiempo de seguimiento de la serie presentada hace necesario que la interpretación de los resultados ofrecidos deba 


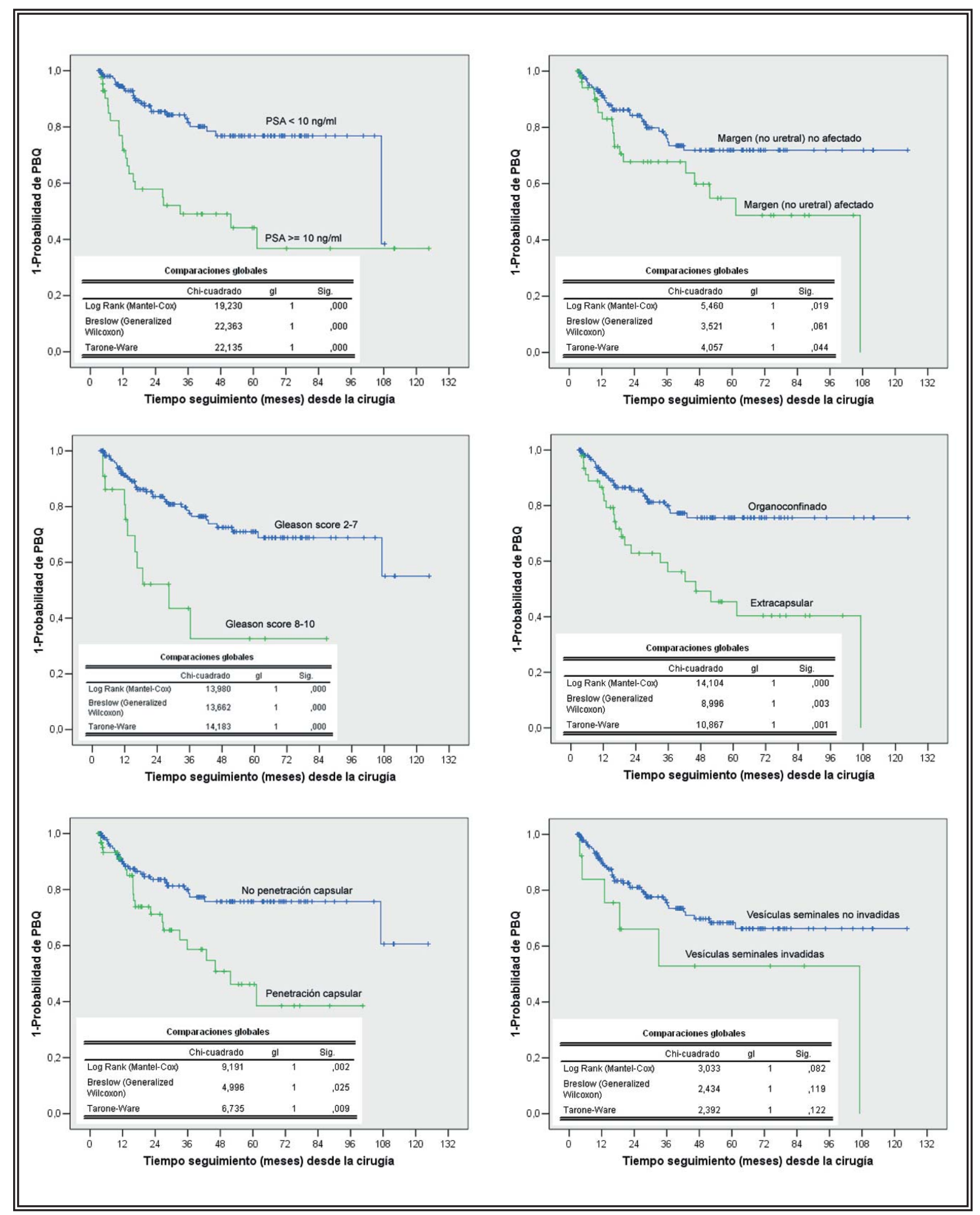

FIGURA 3: Impacto de diversos factores sobre el evento recurrencia de PSA (análisis univariante).

3a) Nivel de PSA preoperatorio. 3b) Score de Gleason de la pieza quirúrgica. 3c) Penetración capsular. 3d) Margen quirúrgico positivo (excluyendo uretral). 3e) Extensión extracapsular. 3f) Invasión de vesículas seminales. 
Tabla 2

Resultado del análisis multivariante (modelo de regresión de Cox) donde se identifican los factores predictores independientes de la aparición de progresión de PSA.

\begin{tabular}{lcccc}
\hline & & & \multicolumn{2}{c}{$\mathbf{9 5 , 0 \%}$ IC para HR } \\
& p & Hazard ratio (HR) & Inferior & Superior \\
\hline PSA <=10 NG/ML &, 000 & 3,03 & 1,66 & 5,55 \\
Gleason score 8-10 &, 001 & 3,42 & 1,61 & 7,26 \\
Penetración capsular &, 012 & 2,17 & 1,18 & 3,97 \\
\hline
\end{tabular}

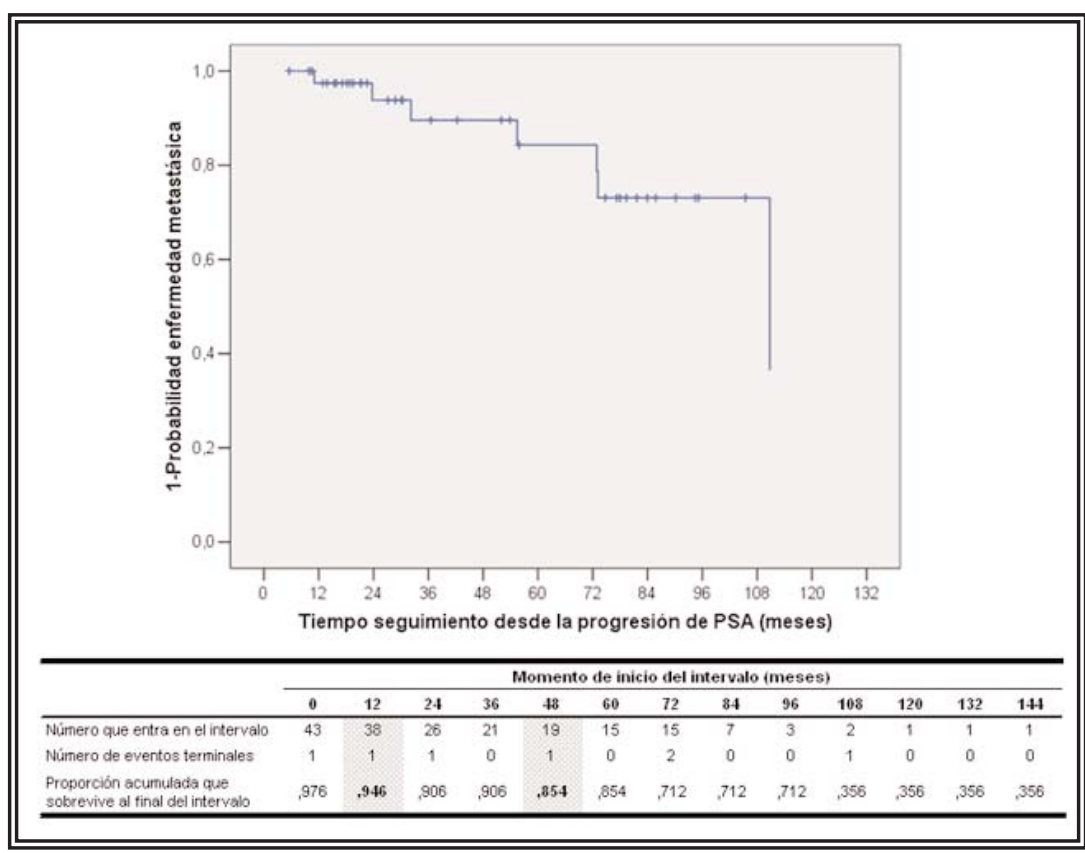

FIGURA 4: Evolución de los pacientes que sufrieron progresión de PSA. Probabilidad de permanecer libre de diseminación a distancia a lo largo del tiempo.

hacerse con precaución, siendo necesario el análisis en un futuro con un mayor tiempo de seguimiento.

Tras el análisis efectuado, tan sólo el presentar un nivel de PSA preoperatorio $>=10$ $\mathrm{ng} / \mathrm{ml}$, un score de Gleason en la pieza entre 8 y 10 (ambos con un riesgo de PBQ superior a 3 veces en caso de presentarse cualquiera de los dos factores), y el hallazgo de penetración capsular han demostrado poseer carácter predictor independiente para la recurrencia de PSA tras la PR. Estos datos (excepto la penetración capsular) han sido también identificados en otras series ${ }^{5}$.

Hemos constatado, del mismo modo que otras series $^{4-6}$, que el mayor riesgo de recurrencia tras la PR se presenta durante los dos primeros años
(Fig. 2). Esto podría sugerir que la PBQ se podría deber a un infraestadiaje, aunque las limitaciones de número y seguimiento de nuestra muestra no han demostrado en el análisis multivariante que la presencia de enfermedad extracapsular produzca una mayor tasa de progresiones.

\section{CONCLUSIONES}

El pronóstico de los pacientes con recurrencia de PSA tras la prostatectomía radical sin recibir tratamiento activo es, en términos de desarrollo de enfermedad metastásica, aceptable a corto y medio plazo. No obstante, será necesario un nuevo análisis con mayor tiempo de seguimiento para confirmar o rechazar la necesidad de tratamiento precoz en estos pacientes.

\section{Agradecimientos $y$ Soporte financie} ro: Financiado en parte por becas del Fondo de Investigación Sanitaria (FIS) (93/0903, 96/0248, 96/1800, 99/0245 y 02/0732).

Nuestro Centro es miembro participante en el "European Randomized Study of Screening for Prostate Cancer (ERPSC)".

\section{REFERENCIAS}

1. Eastham JA, Scardino PT. Radical prostatectomy. En "Campbell's Urology", pp 3080-3106. 8th Ed. Saunders. Philadelphia PA, USA.

2. Walsh PC. Anatomic radical prostatectomy: Evolution of the surgical technique. J Urol 1998;160:2418-2424.

3. Kaplan EL, Meier P. Nonparametric estimation from incomplete observations. $J$ of the American Statistical Association 1958;53:457-481.

4. Pound CR, Partin AW, Eisenberger MA, et al: Natural history of progression after PSA elevation following radical prostatectomy. JAMA 1999;281:1591-1597.

5. Hull GW, Rabbani F, Abbas F, Wheeler TM, Kattan MW, Scardino PT. Cancer control with radical prostatectomy alone in 1000 consecutive cases. J Urol 2002;167:528-534.

6. Dillioglugil O, Leibman BD, Kattan MW, Seale-Hawkins C, Wheeler TM, Scardino PT. Hazard rates for progression after radical prostatectomy for clinically localized prostate cancer. Urology 1997a;50:93-99.

Dr. M. Luján Galán

Servicio de Urología. Hospital Univ. de Getafe Ctra. Toledo km. 12,500 Getafe - 28905 Madrid E-mail: marcoslujan@terra.es

(Trabajo recibido el 31 de agosto de 2005) 